\title{
Survival and prognostic factors for patients with malignant central airway obstruction following airway metallic stent placement
}

\author{
Hui-Wen Qian ${ }^{1 \#}$, Ping Zhang ${ }^{1,2 \#}$, Xin Wang, ${ }^{3,4 \#}$, Yi Zhang ${ }^{5}$, Juan $\mathrm{Li}^{1}$, En-Jian Zhong ${ }^{1,6}$, Shun-Dong $\mathrm{Ji}^{7}$, \\ Jing $\mathrm{Li}^{1,8}$, Li-Rong Zou ${ }^{1}$, Sheng $\mathrm{Xu}^{4}$, Yong-Hong Zhang ${ }^{9}$, Jian-An Huang ${ }^{1}$, Jin-Ming Yang ${ }^{10}$, \\ Chong-Ke Zhong ${ }^{9}$, Cheng Ji ${ }^{1}$
}

${ }^{1}$ Department of Respiratory and Critical Care Medicine, The First Affiliated Hospital of Soochow University, Suzhou, China; ${ }^{2}$ Department of Respiratory Medicine, The Shangrao People's Hospital, Shangrao, China; ${ }^{3}$ Department of Oncology, Fuzhou Traditional Chinese Medicine Hospital, Fuzhou, China; ${ }^{4}$ Department of Radiotherapy, The First Affiliated Hospital of Soochow University, Suzhou, China; ${ }^{5}$ Department of Pharmacology, College of Pharmaceutical Sciences, Soochow University, Suzhou, China; ${ }^{6}$ Department of Pulmonary and Critical Care Medicine, The First People's Hospital of Kunshan, Kunshan, China; ${ }^{7}$ The First Affiliated Hospital of Soochow University, Jiangsu Institute of Hematology, MOH Key Laboratory of Thrombosis and Hemostasis, Collaborative Innovation Center of Hematology, Suzhou, China; ${ }^{8}$ Department of Ultrasound, The Second Affiliated Hospital of Soochow University, Suzhou, China; ${ }^{9}$ Department of Epidemiology, School of Public Health and Jiangsu Key Laboratory of Preventive and Translational Medicine for Geriatric Diseases, Medical College of Soochow University, Suzhou, China; ${ }^{10}$ Department of Cancer Biology and Toxicology, College of Medicine, University of Kentucky, KY, USA

Contributions: (I) Conception and design: P Zhang, HW Qian, C Ji; (II) Administrative support: None; (III) Collection and assembly of data: P Zhang, HW Qian; (IV) Data analysis and interpretation: HW Qian, P Zhang, X Wang, CK Zhong, C Ji; (V) Manuscript writing: All authors; (VI) Final approval of manuscript: All authors.

\#These authors contributed equally to this work.

Correspondence to: Cheng Ji. Department of Respiratory and Critical Care Medicine, The First Affiliated Hospital of Soochow University, No. 188 Shizi Street, Suzhou 215006, China. Email: jicheng_suda@163.com; Chong-Ke Zhong. Department of Epidemiology, School of Public Health and Jiangsu Key Laboratory of Preventive and Translational Medicine for Geriatric Diseases, Medical College of Soochow University, Suzhou 215123, China. Email: ckzhong@suda.edu.cn.

Background: There have been many studies on the effectiveness and complications of airway stent, but few had focused on factors that affect survival after stent placement. This study intended to assess the factors associated with the survival in patients with malignant central airway obstruction (MCAO) after airway metallic stent placement.

Methods: The clinical data of adult MCAO patients who underwent stent placement form February 2003 to June 2017 in the First Affiliated Hospital of Soochow University in China were retrospectively analyzed. The survival rates were compared using Log-rank tests. Potential prognostic factors were identified using multivariate Cox hazard regression models.

Results: Total 102 MCAO patients were included in this study. The median survival time of these patients after airway metallic stent placement was 4.1 months. Multivariate analysis showed that MCAO patients receiving radiotherapy [hazard ratio (HR) 0.554; $95 \%$ confidence interval (CI): 0.308-0.999] or chemoradiotherapy (HR 0.251; 95\% CI: 0.126-0.499) after stenting had better prognosis. However, ECOG PS $\geq 3$ score prior to the stenting (HR 2.193; 95\% CI: 1.364-3.526) and stents placed in both trachea and main bronchus (HR 2.458; 95\% CI: 1.384-4.366) were associated with worse survival.

Conclusions: In our results, survival of MCAO patients after airway metallic stenting was related to ECOG PS score prior to the stenting, the site of stent placement and we have hereby proposed for the first time that having opportunity to receive radiotherapy or chemoradiotherapy after stenting contribute to better prognosis. 
Keywords: Bronchoscopic interventions; stents; malignant central airway obstruction (MCAO); survival; prognostic factors

Submitted Mar 29, 2020. Accepted for publication Oct 28, 2020.

doi: $10.21037 /$ jtd-20-1520

View this article at: http://dx.doi.org/10.21037/jtd-20-1520

\section{Introduction}

Central airway obstruction (CAO) (1) is defined as a reduced lumen located in the trachea, carina, main bronchi or right bronchus intermedius. It can be caused by a variety of malignant and benign disease, with the former as the most common one (2). Primary pulmonary malignancy, adjacent organ malignancies such as esophageal cancer, thyroid cancer, lymphoma and distant metastatic malignancies, all can cause central airway stenosis through direct intraluminal tumor growth or external compression the airways. Malignant central airway obstruction (MCAO) can lead to airflow limitation, atelectasis, obstructive pneumonia and hemoptysis. Due to the severity of illness, some inoperable MCAO require airway intervention such as laser, electrocautery, balloon dilatation, cryotherapy and with or without stent placement before or during antitumor therapy (3). Because of the rapid and long-lasting effect of alleviating airway obstruction, stenting is often the preferred choice for patients with inoperable lifethreatening MCAO, especially for the type of extrinsic airway compression (4).

So far, there have been many studies on the effectiveness and complications of airway stenting, but few had focused on factors that affect survival after stenting $(5,6)$. Nagano et al. reported that a good performance status was the only positive factor associated with survival after airway stenting, but this study only analyzed 21 patients (7). At present, what should be done for the MCAO patients following stent placement remains ambiguous? Are there any other factors can affect the survival? Therefore, we aimed to assess the factors associated with the survival in a Chinese population with MCAO after stent placement.

\section{Methods}

\section{Patients population}

All patients who underwent stent placement because of MCAO from February 2003 to June 2017 in the First Affiliated Hospital of Soochow University (a 2,500-bed, a general hospital in Suzhou, China) were included in this study. Clinical data and follow-up information were obtained from medical records or through telephone interviews with the patients' families. Criteria used to recruit the patients to this study included: (I) CAO were identified by bronchoscope or chest computed tomography (CT) with cross-sectional area of lumen with decrease of more than $50 \%$, or with obvious dyspnea symptoms; (II) airway stenosis was caused by malignant tumor; and (III) the patients received airway stent placement. Exclusion criteria were: (I) stent was placed because of esophagotracheal fistula but not MCAO; (II) clinical data was incomplete (Figure 1).

Data of each patient were collected including baseline data [sex, age, body mass index (BMI), smoking history, and basic comorbidities], clinical features (etiology, differentiation, stage, treatment before stenting, and ECOG PS score), laboratory test data [neutrophil to lymphocyte ratio (NRL), hemoglobin (Hb), albumin (ALB), and serum lactate dehydrogenase (LDH)], characteristics of stenosis, stent placement (location, type and complications), treatment and survival after stenting.

The study was conducted in accordance with the Declaration of Helsinki (as revised in 2013). This study was approved by the ethics committee of the First Affiliated Hospital of Soochow University (2019-009). The informed consensus from each patient or the individual patient's family was waived because there were no new interventions for the patients and the information was anonymized.

\section{Definition}

ECOG PS scores were assessed before stenting according to the literature (8). The severity of CAO was assessed according to the proposed criteria (9): degree 0 : normal; degree I: reduction of cross-sectional area $\leq 25 \%$; degree II: reduction of cross-sectional area $>25 \%$ but $\leq 50 \%$; degree III: reduction of cross-section area $>50 \%$ but $\leq 75 \%$; degree IV: reduction of cross-sectional area $>75 \%$ but $\leq 90 \%$; and degree $\mathrm{V}$ : reduction of cross-sectional area $>90 \%$. 


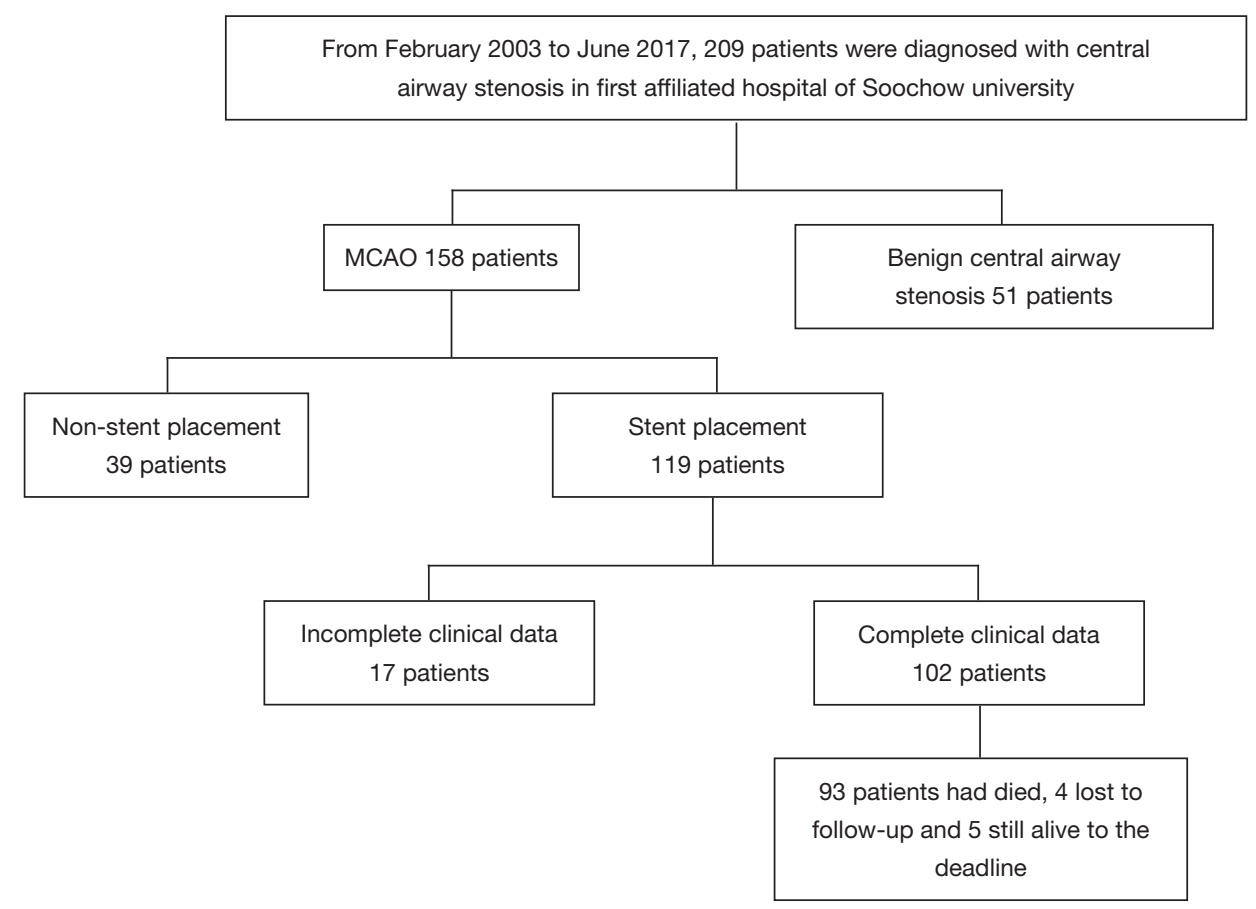

Figure 1 Flow chart of the recruitment of those patients in the study.

\section{Airway stenting}

\section{Preoperative setup}

Prior to the stenting, all patients underwent chest CT with or without a flexible bronchoscope exam to assess the severity of CAO.

\section{Procedure}

After local or general anesthesia, the stent (straight, L or Y-shaped metallic airway stent) was guided by flexible bronchoscope or through endotracheal intubation under $\mathrm{X}$-ray. If airway was significantly obstructed, airway intervention such as balloon dilatation would be performed before stenting. Due to the limitations of technology and materials, we did not carry out silicone stent placement. Instead, we used metallic airway stent mainly manufactured by Micro-Tech (Nanjing) Co., Ltd.

\section{Follow up}

Routine bronchoscopy would be performed one week after stenting to check the status of the stent and tenacious secretions. Subsequent bronchoscopy was determined by the attending physician if necessary. After the initial stenting, bronchoscopic interventions to maintain airway patency including argon plasma coagulation (APC), laser, stent replacement, cryotherapy, etc. would be performed if recurrence of $\mathrm{CAO}$ caused by tumor, granulation tissue formation or tenacious secretions.

\section{Statistical analysis}

Continuous variables were expressed as mean \pm SD and categorical variables were expressed as frequency (\%). Survival length was calculated from the time of the stent insertion to the time of death or last follow-up if the patients were still alive (June 2017, last follow up). The overall survival rate was presented by the Kaplan-Meier curve. The Log-rank test was used to compare survival rates of subgroups of each potential prognostic factor. Then factors with $\mathrm{P}$ value of less than 0.05 based on Log-rank analyses or may affect prognosis were analyzed by multivariate Cox hazards regression model using forward stepwise regression based on maximum likelihood estimation. Hazard ratio (HR) and 95\% confidence interval (CI) were calculated for potential predictors. All of the $\mathrm{P}$ values were two-tailed, and a significance level of 0.05 was used. Statistical analysis was conducted using SPSS statistical software (SPSS, version 22.0, Chicago, IL, USA). 
Table 1 The baseline characteristic of 102 patients with MCAO

\begin{tabular}{|c|c|}
\hline Variables & Total $(n=102)$ \\
\hline Male & $67(65.7 \%)$ \\
\hline Age (years) & $64 \pm 10.0[28-88]$ \\
\hline Body mass index $\left(\mathrm{kg} / \mathrm{m}^{2}\right)$ & $21.4 \pm 3.0(15.1-28.9)$ \\
\hline Smoking & $56(54.9 \%)$ \\
\hline \multicolumn{2}{|l|}{ Primary disease } \\
\hline Lung cancer & $32(31.4 \%)$ \\
\hline Esophageal cancer & $49(48.0 \%)$ \\
\hline Thyroid cancers & $11(10.8 \%)$ \\
\hline Unknown mediastinal malignancies & $3(2.9 \%)$ \\
\hline Thymic carcinoma & $1(1.0 \%)$ \\
\hline Laryngeal carcinoma & $2(2.0 \%)$ \\
\hline Rectal cancer & $2(2.0 \%)$ \\
\hline Trachea adenoid cystic carcinoma & $1(1.0 \%)$ \\
\hline Nasopharyngeal carcinoma & $1(1.0 \%)$ \\
\hline \multicolumn{2}{|l|}{ Basic comorbidities } \\
\hline Hypertension & $45(44.1 \%)$ \\
\hline Diabetes mellitus & $12(11.8 \%)$ \\
\hline Coronary disease & $2(2.0 \%)$ \\
\hline Chronic lung disease & $11(10.8 \%)$ \\
\hline Cerebrovascular disease & $6(5.9 \%)$ \\
\hline Connective tissue disease & $3(2.9 \%)$ \\
\hline Others & $9(8.8 \%)$ \\
\hline \multicolumn{2}{|l|}{ Clinical manifestation } \\
\hline Dyspnea & $86(84.3 \%)$ \\
\hline Cough & $56(54.9 \%)$ \\
\hline Hemoptysis & $27(26.5 \%)$ \\
\hline Fever & $3(2.9 \%)$ \\
\hline \multicolumn{2}{|l|}{ Respiratory sounds } \\
\hline Inspiratory wheezing & $26(25.5 \%)$ \\
\hline Weakening & $20(19.6 \%)$ \\
\hline \multicolumn{2}{|l|}{ Pathological stage } \\
\hline III & $18(17.6 \%)$ \\
\hline IV & $84(82.4 \%)$ \\
\hline \multicolumn{2}{|l|}{ Pathological differentiation } \\
\hline undifferentiated or low & $39(38.2 \%)$ \\
\hline middle - high & $63(61.8 \%)$ \\
\hline \multicolumn{2}{|l|}{ ECOG PS score } \\
\hline $0-2$ & $36(35.3 \%)$ \\
\hline $3-4$ & $66(64.7 \%)$ \\
\hline
\end{tabular}

\section{Results}

\section{Clinical data and survival}

Total 102 consecutive patients who underwent airway stent placement due to MCAO were included in this study. Characteristics of baseline were summarized in Table 1 . These patients aged from 28 to 88 years old with a mean age of $64 \pm 10.0$ years old, and the male/female ratio was 67:35. BMI ranged from 15.1 to $28.9 \mathrm{~kg} / \mathrm{m}^{2}$ with an average of $21.4 \mathrm{~kg} / \mathrm{m}^{2}$. Fifty-six patients $(54.9 \%)$ had a history of smoking. Esophageal cancer (49/102, 48.0\%) and lung cancer $(32 / 102,31.4 \%$, including 4 cases of small cell lung cancer) were the main common diseases that caused MCAO in this study, followed by thyroid carcinoma (11/102, $10.8 \%$, including 9 cases of papillary carcinoma, 1 case of follicular carcinoma, and only 1 case of undifferentiated carcinoma) and other malignant tumors (10/102, 9.8\%). The basic comorbidities were hypertension (44.1\%), diabetes $(11.8 \%)$, coronary heart disease $(2.0 \%)$, chronic lung disease $(10.8 \%)$, cerebrovascular disease $(5.9 \%)$, connective tissue disease (2.9\%) and other diseases (8.8\%). The main clinical symptoms were dyspnea (84.3\%), cough (54.9\%), hemoptysis (26.5\%) and fever (2.9\%). Physical examinations of respiratory sounds revealed 26 cases (25.5\%) with inspiratory wheezing. The pathology stage of 18 cases $(17.6 \%)$ were stage III and 84 cases $(82.4 \%)$ were stage $\mathrm{IV}$ at the time when diagnosed as MCAO. 39 patients $(38.2 \%)$ were caused by low or undifferentiated tumors and the others $(61.8 \%)$ were caused by middle or high differentiated tumors.

A total of 120 metallic stents were implanted in these patients, 1 to 3 stents per patient. Five patients received subsequent re-stenting due to recurrence of CAO. Characteristics of the MCAO were summarized in Table 2. Most of stenting were performed under local anesthesia (65.7\%). Carinal involvement was founded in 45 cases (44.1\%). Stenosis was caused by intraluminal tumor growth in 4 cases (3.9\%), external compression in 29 cases $(28.4 \%)$ and mixed in 69 cases $(67.6 \%)$. The degree of stenosis was grade II in 6 cases $(5.9 \%)$, grade $\mathrm{III}$ in 31 cases $(30.4 \%)$, grade IV in 49 cases $(48.0 \%)$ and grade $\mathrm{V}$ in 16 cases $(15.7 \%)$. The site of stent placement only in trachea was 71 cases $(69.6 \%)$.

Shortness of breath was rapidly relieved in most of patients with MCAO after stenting. One patient needed non-invasive assisted ventilation after stenting. The complications after stenting (shown in Table S1) included bloody sputum (26.5\%), irritating cough (17.6\%), foreign body sensation in pharynx $(13.7 \%)$, stent fracture $(2.0 \%)$, 
Table 2 The characteristics of the first airway stent placement in 102 MCAO patients

\begin{tabular}{|c|c|}
\hline Variables & Total $(n=102)$ \\
\hline \multicolumn{2}{|l|}{ Anesthesia method } \\
\hline General & $35(34.3 \%)$ \\
\hline Local & $67(65.7 \%)$ \\
\hline Invasive carina & $45(44.1 \%)$ \\
\hline \multicolumn{2}{|l|}{ Degree of stenosis } \\
\hline II & $6(5.9 \%)$ \\
\hline III & $31(30.4 \%)$ \\
\hline IV & $49(48.0 \%)$ \\
\hline V & $16(15.7 \%)$ \\
\hline \multicolumn{2}{|l|}{ Type of stenosis } \\
\hline Internal tumor growth & $4(3.9 \%)$ \\
\hline External compression & $29(28.4 \%)$ \\
\hline Mixed & $69(67.6 \%)$ \\
\hline \multicolumn{2}{|l|}{ Site of stents } \\
\hline Trachea & $71(69.6 \%)$ \\
\hline Left main bronchus & $6(5.9 \%)$ \\
\hline Right main or intermedius bronchus & $5(4.9 \%)$ \\
\hline Right main + left main bronchus & $2(2.0 \%)$ \\
\hline Trachea + right main bronchus & $5(4.9 \%)$ \\
\hline Trachea + left main bronchus & $6(5.9 \%)$ \\
\hline $\begin{array}{l}\text { Trachea + left main bronchus + right main } \\
\text { bronchus }(Y)\end{array}$ & $7(6.9 \%)$ \\
\hline
\end{tabular}

$\mathrm{MCAO}$, malignant central airway obstruction.

stent migration (3.9\%), tracheoesophageal fistula (4.9\%), restenosis $(29.4 \%)$ (caused by tumor (16.7\%), granulation tissue formation (6.9\%), and tenacious secretions (5.9\%). 22 patients $(21.6 \%)$ underwent bronchoscopic interventions including electrocautery, APC, cryotherapy, tenacious secretion clearance (6 cases) and stent replacement (4 cases) because of re-obstruction. After stenting, 57 cases (55.9\%) received only supportive treatment, 19 cases (18.6\%) received radiotherapy (including external radiotherapy, endobronchial brachytherapy and radioactive particle implantation therapy and two thyroid cancer patients were treated with isotope iodine 131-a special radiotherapy), 11 cases $(10.8 \%)$ received systemic chemotherapy, and 15 cases (14.7\%) received chemoradiotherapy.
By the end of this study, 93 patients had died, 4 patients lost follow-up, and 5 patients were still alive. The median survival time was 4.1 months. The survival rates at 1, 3, 6, 9 and 12 months were $93.1 \%, 63.7 \%, 32.4 \%, 15.7 \%$ and $4.9 \%$, respectively. The overall survival curve was showed in Figure S1.

\section{Univariate analysis of survival by log-rank test}

Univariate analysis of 20 potential prognostic factors in the 102 cases showed that pathological staging, history of chemotherapy or radiotherapy prior to the stenting procedure, ECOG PS score prior to the stenting procedure, NRL, carinal involvement, site of stent placement and postprocedural treatments were associated with the survival in MCAO patients $(\mathrm{P}<0.05)$. While, factors such as tumor differentiation, degree and type of stenosis were not associated with the prognosis (Table 3). The survival curves using Kaplan-Meier estimates were showed in Figure 2.

\section{Multivariate analysis of survival by Cox regression}

Factors associated with the survival of MCAO patients after airway stenting, as revealed by univariate analysis $(\mathrm{P}<0.05)$, and others mentioned in the previous studies or thought to have an impact on prognosis were further analyzed using the multivariate Cox hazards regression model. Multivariate analysis showed that ECOG PS score prior to the stenting procedure, site of stent placement and post-procedural treatments were significantly associated with survival (Table 4). Patients with high ECOG PS score prior to the stenting (3 to 4 score) had worse prognosis as compare to those with low ECOG PS (0 to 2 score) (HR 2.193; 95\% CI: 1.364 3.526; $\mathrm{P}=0.001$ ). Patients with stent placed in both trachea and main bronchus had worse prognosis than those with stent placed only in trachea (HR 2.458; 95\% CI: 1.384-4.366; $\mathrm{P}=0.002)$. No difference was found between patients with stents only placed in trachea and those placed in main bronchus (HR 1.080; 95\% CI: 0.564-2.070; P=0.816). After stenting, patients who had chances to receive radiotherapy (HR 0.554; 95\% CI: 0.308-0.999; $\mathrm{P}=0.049628$ ) or chemoradiotherapy (HR 0.251; 95\% CI: $0.126-0.499 ; \mathrm{P}<0.001)$ had better prognosis than those who only received supportive therapy.

\section{Discussion}

MCAO are associated with poor survival and the median survival of those untreated patients was only 1-2 months (10). 
Table 3 The result of Log-Rank analyses of $102 \mathrm{MCAO}$ patients after stenting

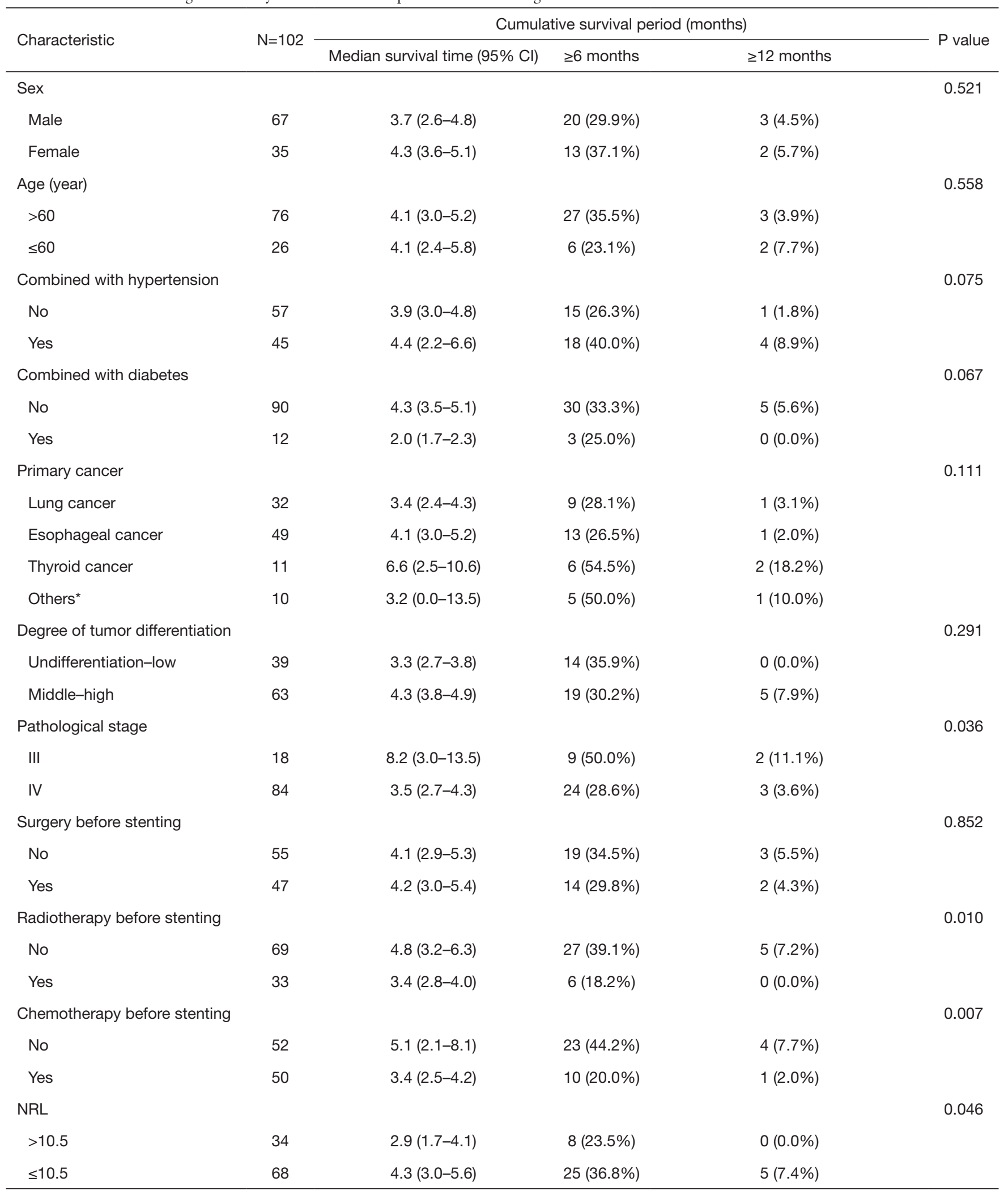

Table 3 (continued) 
Table 3 (continued)

\begin{tabular}{|c|c|c|c|c|c|}
\hline Characteristic & $\mathrm{N}=102$ & \multicolumn{3}{|c|}{ Cumulative survival period (months) } & $P$ value \\
\hline $\mathrm{Hb}(\mathrm{g} / \mathrm{L})$ & & & & & 0.092 \\
\hline$\geq 110$ & 66 & $4.3(2.7-5.9)$ & $24(36.4 \%)$ & $5(7.6 \%)$ & \\
\hline$<110$ & 36 & $3.3(1.7-4.9)$ & $9(25.0 \%)$ & $0(0.0 \%)$ & \\
\hline$\geq 40$ & 36 & $3.5(1.5-5.6)$ & $12(33.3 \%)$ & $2(5.6 \%)$ & \\
\hline$<40$ & 66 & $4.1(3.2-5.0)$ & $21(31.8 \%)$ & $3(4.5 \%)$ & \\
\hline LDH level (u/L) & & & & & 0.058 \\
\hline$\leq 250$ & 76 & $4.4(3.4-5.4)$ & $28(36.8 \%)$ & $4(5.3 \%)$ & \\
\hline III & 31 & $4.8(0.5-9.0)$ & $14(45.2 \%)$ & $0(0.0 \%)$ & \\
\hline IV & 49 & $4.2(2.6-5.8)$ & $13(26.5 \%)$ & $2(4.1 \%)$ & \\
\hline V & 16 & $3.3(0.7-5.8)$ & $5(31.3 \%)$ & $2(12.5 \%)$ & \\
\hline Type of stenosis & & & & & 0.337 \\
\hline Internal tumor growth & 4 & $4.4(0.0-8.8)$ & $1(25.0 \%)$ & $1(25.0 \%)$ & \\
\hline External compression & 29 & $5.0(3.3-6.7)$ & $12(41.4 \%)$ & $1(3.4 \%)$ & \\
\hline Mixed & 69 & $3.5(2.6-4.4)$ & $20(29.0 \%)$ & $3(4.3 \%)$ & \\
\hline Main bronchus & 13 & $3.7(1.9-5.5)$ & $3(23.1 \%)$ & $1(7.7 \%)$ & \\
\hline Trachea + main bronchus & 18 & $1.8(1.4-2.2)$ & $2(11.1 \%)$ & $0(0.0 \%)$ & \\
\hline ECOG PS score before stenting & & & & & $<0.001$ \\
\hline $0-2$ & 36 & $7.8(5.7-9.9)$ & $20(55.6 \%)$ & $4(11.1 \%)$ & \\
\hline $3-4$ & 66 & $3.0(2.6-3.4)$ & $13(19.7 \%)$ & $1(1.5 \%)$ & \\
\hline Post-procedure treatment & & & & & $<0.001$ \\
\hline Support treatment & 57 & $2.9(1.9-3.9)$ & $9(15.8 \%)$ & $1(1.8 \%)$ & \\
\hline Radiotherapy & 19 & $5.5(4.9-6.1)$ & $7(36.8 \%)$ & $0(0.0 \%)$ & \\
\hline Systemic chemotherapy & 11 & $3.4(1.0-5.7)$ & $4(36.4 \%)$ & $1(9.1 \%)$ & \\
\hline Chemoradiotherapy & 15 & $9.5(7.8-11.2)$ & $13(86.7 \%)$ & $3(20.0 \%)$ & \\
\hline
\end{tabular}

${ }^{*}$ Others: other primary cancers include 3 unknown mediastinal malignancies, 1 thymic carcinoma, 2 laryngeal carcinomas, 2 rectal cancers, 1 trachea adenoid cystic carcinoma and 1 nasopharyngeal carcinoma. MCAO, malignant central airway obstruction. 
A

ECOG PS score before stenting

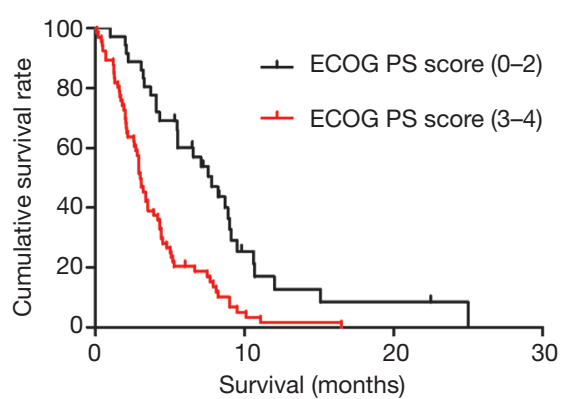

C

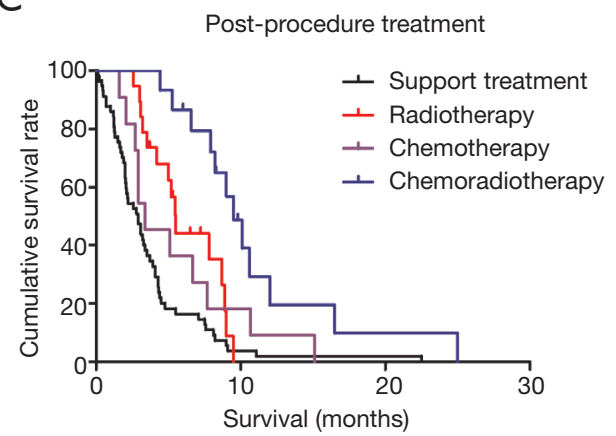

B Site of stent placement

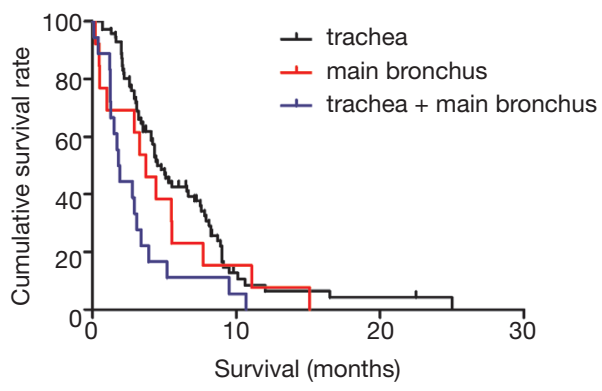

Figure 2 Overall survival curves using Kaplan-Meier estimates. Survival rates based on (A) ECOG PS score before stenting $(\mathrm{P}<0.001)$, (B) site of stent placement $(\mathrm{P}=0.005)$, (C) post-procedure treatments $(\mathrm{P}<0.001)$.

Table 4 The result of Cox regression analysis of 102 MCAO patients after stenting

\begin{tabular}{lcc}
\hline Characteristic & HR & $95 \% \mathrm{Cl}$ \\
\hline ECOG PS score before stenting & 1 & Reference \\
$0-2$ & 2.193 & $1.364-3.526$ \\
$3-4$ & & 0.001 \\
Site of stent placement & 1 & Reference \\
Trachea & 1.080 & $0.564-2.070$ \\
Main bronchus & 2.458 & $1.384-4.366$ \\
Trachea + main bronchus & & 0.816 \\
Post-procedure treatment & 1 & 0.002 \\
Support treatment & 0.554 & $0.308-0.999$ \\
Radiotherapy & 0.507 & $0.252-1.023$ \\
Chemotherapy & 0.251 & $0.126-0.499$ \\
Chemoradiotherapy & & 0.058 \\
\hline
\end{tabular}

${ }^{*} \mathrm{P}=0.049628$. MCAO, malignant central airway obstruction. 
With the development of bronchoscopic interventions technique, the CAO can be alleviated by these approaches, thus these patients have opportunity to receive further antitumor treatment to prolong their life $(11,12)$. In our study, the median survival of MCAO patients after stenting was 4.1 months, similar to reported by Guibert et al. (4.7 months) (5) and Tanigawa et al. (3.6 months) (13).

Interventional bronchoscopy is considered as one of the positive prognostic factors in patients with MCAO (14). In addition, it has been reported that etiology, ASA score, obstruction site, extrinsic or mixed lesion, preoperative adjuvant therapy, preoperative ECOG PS score and postoperative treatment were independent prognosisrelated factors in MACO patients receiving interventional bronchoscopy $(5,6,15-17)$. Compared to the above studies, our study focused on the MCAO patients treated with stenting, which excluding the influence of other interventions. Our study showed that ECOG PS score before stenting, site of stent placement and post-procedural treatments were the independent prognostic factors for the patients with MCAO after airway stenting.

ECOG PS score is an indication that helps determine whether a cancer patient should receive chemotherapy and is also a prognostic factor of cancer patients $(18,19)$. Similar to reported by Razi et al. (20), our study showed that patients with high PS score [3-4] had a worse prognosis than those with low PS score [0-2] (median survival 3.0 vs. 7.8 months, $\mathrm{P}=0.001$ ). Indeed, symptoms of dyspnea or hypoxia could be partially alleviated after stenting, but whether the tumor could be controlled or not depends on subsequent anti-tumor treatment. Patients with high PS score may have fewer chances to receive the following antitumor treatment, which may lead to worse prognosis.

Dalar et al. reported that the lesion site was one of independent predictors of survival in MCAO patients. The type (straight, L or Y- shaped) and length of airway stent are also determined by the sites of the lesion (15). Our study showed that patients who placed stent both in the trachea and main bronchus had worse prognosis as compared to the patients who only placed in trachea (median survival: $1.8 \mathrm{vs}$. 4.5 months; $\mathrm{P}=0.002$ ). Nevertheless, there was no difference between the groups of the stent placement in trachea and main bronchus. This reflects the complex MCAO (stenosis involved both in the trachea and main bronchus) is more severe than the simple MCAO (stenosis only involved in the trachea or main bronchus).

Song et al. showed that anti-tumor treatment prolonged the survival of the patients with MCAO after receiving bronchoscopic intervention $(\mathrm{P}<0.001)$ (6) and Jeon et al. reported the same conclusion $(\mathrm{P}<0.001)(21)$. But none of them indicated the effect of different anti-tumor therapies on prognosis of these MCAO patients after bronchoscopic intervention. Our data extended these information and showed the median survival of the group receiving radiotherapy and chemoradiotherapy treatment after stenting was 5.5 and 9.5 months, respectively, which were more than 2.9 months of supportive treatment group $(\mathrm{P}<0.05, \mathrm{P}<0.001$; respectively). But no difference was found between the chemotherapy group after stenting and the supportive treatment group $(\mathrm{P}>0.05)$. This could be partly explained by most of cases were at the end-stage and nearly one-half of patients had received chemotherapy before stenting. As a means of local treatment, radiotherapy is more effective than chemotherapy in controlling local airway stenosis. Our data shows that patients with MCAO who receive radiotherapy or radiochemotherapy after stenting have a longer survival time, which suggests that radiotherapy as a local treatment to maintain airway patency is more important for those patients, especially at the endstage or previously received chemotherapy.

It had been previously reported that the type of primary tumor was one of the prognostic factors in patients with MCAO after bronchoscopic intervention (14). However, in our study, we didn't find the difference of survival between different tumor types after airway stenting. This may be caused by different patients participating in the study.

The study also has some limitations, including: (I) it was a single-center, retrospective study; (II) different tumors responded differently to radiotherapy and chemotherapy. Moreover, isotope iodine 131 treatment is not equivalent to radiotherapy (Isotope iodine 131 treatment is accepted as a special kind of radiotherapy in this study). Therefore, different patients enrolled in the study may affect the outcome; (III) none of the patients received targeted therapy or immunotherapy, which limited analysis of the impact of these new treatment on the survival; (IV) due to the limitations of technology, we did not carry out silicone stent airway implantation in the past time, thus we couldn't analyze the effects of different kinds of stents on survival.

\section{Conclusions}

This study shows that the median survival of MCAO patients was 4.1 months after airway metallic stenting. Survival of MCAO patients after airway stenting was related to ECOG PS score prior to the stenting and the site of stent placement, 
and we have hereby proposed for the first time that having opportunity to receive radiotherapy or chemoradiotherapy after stenting contribute to better prognosis.

\section{Acknowledgments}

We would like to be grateful to Prof. Hao-Dong Xu (Department of Pathology, University of Washington Medical Center, Seattle, WA 98195, USA) for critical reading of this manuscript.

Funding: This study was funded by grants from Natural Science Foundation of Jiangsu Province of China (BK20151209) to Cheng Ji; National Natural Sciences Foundation of China (81773749) to Yi Zhang, (81472191) to Shun-Dong Ji.

\section{Footnote}

Data Sharing Statement: Available at http://dx.doi. org/10.21037/jtd-20-1520

Conflicts of Interest: All authors have completed the ICMJE uniform disclosure form (available at http://dx.doi. org/10.21037/jtd-20-1520). The authors have no conflicts of interest to declare.

Ethical Statement: The authors are accountable for all aspects of the work in ensuring that questions related to the accuracy or integrity of any part of the work are appropriately investigated and resolved. The study was conducted in accordance with the Declaration of Helsinki (as revised in 2013). This study was approved by the ethics committee of the First Affiliated Hospital of Soochow University (2019-009). The informed consensus from each patient or the individual patient's family was waived because there were no new interventions for the patients and the information was anonymized.

Open Access Statement: This is an Open Access article distributed in accordance with the Creative Commons Attribution-NonCommercial-NoDerivs 4.0 International License (CC BY-NC-ND 4.0), which permits the noncommercial replication and distribution of the article with the strict proviso that no changes or edits are made and the original work is properly cited (including links to both the formal publication through the relevant DOI and the license). See: https://creativecommons.org/licenses/by-nc-nd/4.0/.

\section{References}

1. Ernst A, Feller-Kopman D, Becker HD, et al. Central airway obstruction. Am J Respir Crit Care Med 2004;169:1278-97.

2. Husain SA, Finch D, Ahmed M, et al. Long-term follow-up of ultraflex metallic stents in benign and malignant central airway obstruction. Ann Thorac Surg 2007;83:1251-6.

3. Mudambi L, Miller R, Eapen GA. Malignant central airway obstruction. J Thorac Dis 2017;9:S1087-110.

4. Dasgupta A, Dolmatch BL, Abi-Saleh WJ, et al. Selfexpandable metallic airway stent insertion employing flexible bronchoscopy: preliminary results. Chest 1998;114:106-9.

5. Guibert N, Mazieres J, Lepage B, et al. Prognostic factors associated with interventional bronchoscopy in lung cancer. Ann Thorac Surg 2014;97:253-9.

6. Song JU, Park HY, Kim H, et al. Prognostic factors for bronchoscopic intervention in advanced lung or esophageal cancer patients with malignant airway obstruction. Ann Thorac Med 2013;8:86-92.

7. Nagano H, Kishaba T, Nei Y, et al. Indications of airway stenting for severe central airway obstruction due to advanced cancer. PLoS One 2017;12:e0179795.

8. Oken MM, Creech RH, Tormey DC, et al. Toxicity and response criteria of the Eastern Cooperative Oncology Group. Am J Clin Oncol 1982;5:649-55.

9. Freitag L, Ernst A, Unger M, et al. A proposed classification system of central airway stenosis. Eur Respir J 2007;30:7-12.

10. Macha HN, Becker KO, Kemmer HP. Pattern of failure and survival in endobronchial laser resection. A matched pair study. Chest 1994;105:1668-72.

11. Chen $\mathrm{CH}, \mathrm{Wu} \mathrm{BR}$, Cheng WC, et al. Interventional pulmonology for patients with central airway obstruction: An 8-year institutional experience. Medicine (Baltimore) 2017;96:e5612.

12. Chhajed PN, Baty F, Pless M, et al. Outcome of treated advanced non-small cell lung cancer with and without central airway obstruction. Chest 2006;130:1803-7.

13. Tanigawa N, Kariya S, Komemushi A, et al. Metallic stent placement for malignant airway stenosis. Minim Invasive Ther Allied Technol 2012;21:108-12.

14. Shin B, Chang B, Kim H, et al. Interventional bronchoscopy in malignant central airway obstruction by extra-pulmonary malignancy. BMC Pulm Med 2018;18:46. 
15. Dalar L, Ozdemir C, Abul Y, et al. Therapeutic bronchoscopic interventions for malignant airway obstruction: A retrospective study from experience on 547 patients. Medicine (Baltimore) 2016;95:e3886.

16. Oki M, Saka H. Airway stenting for patients with airway stenosis because of small cell lung cancer. Clin Respir J 2018;12:2257-63.

17. Kim BG, Shin B, Chang B, et al. Prognostic factors for survival after bronchoscopic intervention in patients with airway obstruction due to primary pulmonary malignancy. BMC Pulm Med 2020;20:54.

18. Ettinger DS, Wood DE, Aisner DL, et al. Non-Small Cell Lung Cancer, Version 5.2017, NCCN Clinical Practice Guidelines in Oncology. J Natl Compr Canc Netw

Cite this article as: Qian HW, Zhang P, Wang X, Zhang Y, Li J, Zhong EJ, Ji SD, Li J, Zou LR, Xu S, Zhang YH, Huang JA, Yang JM, Zhong CK, Ji C. Survival and prognostic factors for patients with malignant central airway obstruction following airway metallic stent placement. J Thorac Dis 2021;13(1):39-49. doi: $10.21037 /$ jtd-20-1520
2017;15:504-35.

19. Reck M, Thatcher N, Smit EF, et al. Baseline quality of life and performance status as prognostic factors in patients with extensive-stage disease small cell lung cancer treated with pemetrexed plus carboplatin vs. etoposide plus carboplatin. Lung Cancer 2012;78:276-81.

20. Razi SS, Lebovics RS, Schwartz G, et al. Timely airway stenting improves survival in patients with malignant central airway obstruction. Ann Thorac Surg 2010;90:1088-93.

21. Jeon K, Kim H, Yu CM, et al. Rigid bronchoscopic intervention in patients with respiratory failure caused by malignant central airway obstruction. J Thorac Oncol 2006;1:319-23. 


\section{Supplementary}

Table S1 Complications of airway stenting.

\begin{tabular}{lc}
\hline Complications & Occurrence \\
\hline Bloody sputum & $26.5 \%$ \\
Irritating cough & $17.6 \%$ \\
Pharyngeal foreign body sensation & $13.7 \%$ \\
Stent fracture & $2.0 \%$ \\
Stent migration & $3.9 \%$ \\
Tracheoesophageal fistula & $4.9 \%$ \\
Restenosis & $29.4 \%$ \\
Tumor & $16.7 \%$ \\
Granulation tissue formation & $6.9 \%$ \\
Tenacious secretions & $5.9 \%$ \\
\hline
\end{tabular}

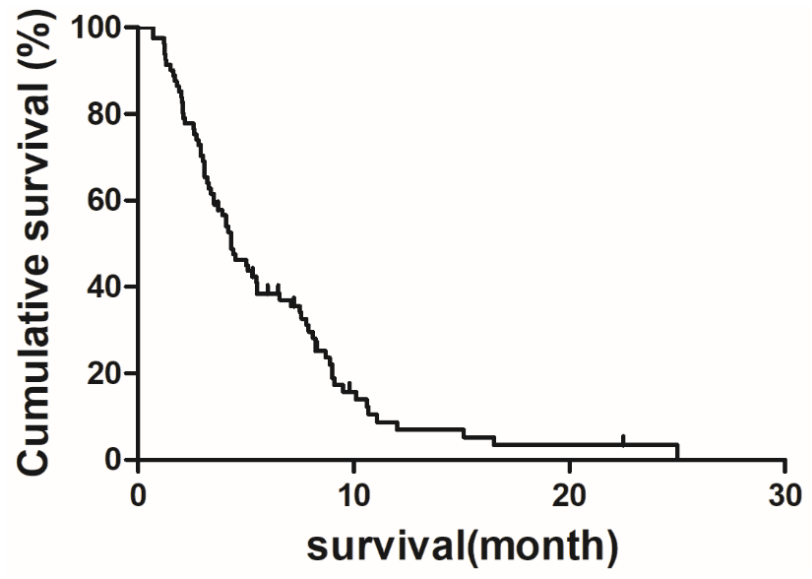

Figure S1 The overall survival rate expressed by the Kaplan-Meier curve. 